\title{
Effect of leg vascular occlusion on arm cycling peak oxygen uptake in spinal cord-injured individuals
}

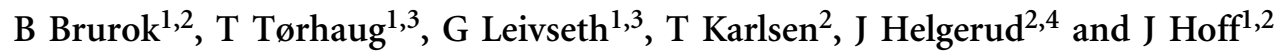

Study design: Cross-sectional single-subject design.

Objectives: To determine whether leg vascular occlusion (LEVO) augment arm cycling (ACE) peak oxygen uptake in spinal cord-injured individuals.

Setting: University Hospital, Norway.

Methods: Fifteen individuals with $\mathrm{C}_{4}$ to $\mathrm{T}_{12}$ spinal cord injury ( $\mathrm{SCl}$ ) were recruited and divided into two groups: injury above (SCl-high, $n=8$ ) or below (SCI-low, $n=7$ ) the $\mathrm{T}_{6}$ level. Peak oxygen uptake $\left(\mathrm{VO}_{2 \text { peak }}\right)$ was measured during (1) ACE combined with LEVO, (2) ACE alone and (3) ACE combined with functional electrical stimulation cycling (FES hybrid cycling).

Results: In the SCl-high group, $\mathrm{VO}_{2 \text { peak }}$ and peak Watt during ACE with LEVO were higher than ACE alone: 20.0 ( \pm 5.0 ) versus 17.6 $( \pm 5.0) \mathrm{ml} \mathrm{kg}^{-1} \mathrm{~min}^{-1}(P=0.006)$, and $72.5( \pm 32)$ versus $80.0( \pm 34)$ Watt $(P=0.016)$, respectively. However, FES hybrid cycling $\mathrm{VO}_{2 \text { peak }}$ was significantly higher than ACE with LEVO: $24.4( \pm 4.1)$ versus $20.0( \pm 5.0) \mathrm{ml} \mathrm{kg}^{-1} \mathrm{~min}^{-1}(P=0.006)$. In the SCl-low group, there was no difference in $\mathrm{VO}_{2 \text { peak }}$ and related parameters between the three modalities.

Conclusions: For spinal cord-injured individuals with injury level above $\mathrm{T}_{6}$ (high) in the present study, LEVO combined with ACE augment $\mathrm{VO}_{2 \text { peak }}$. However, this acute increase in $\mathrm{VO}_{2 \text { peak }}$ was lower than when FES cycling was combined with ACE. These findings may have future implications for exercise prescription for spinal cord-injured individuals.

Spinal Cord (2012) 50, 298-302; doi:10.1038/sc.2011.129; published online 29 November 2011

Keywords: leg vascular occlusion; functional electrical stimulation; arm cycling; peak oxygen uptake

\section{INTRODUCTION}

Persons with spinal cord injury (SCI) have limited aerobic capacity, which is closely linked to increased prevalence of cardiovascular disease. ${ }^{1}$ The limited aerobic capacity in SCI is due to the inability to redistribute blood during arm cycling (ACE). Lack of normal activation of the muscle venous pump (although there is controversy regarding the mechanism behind the effect of the muscle pump in venous return, because vasodilatation is also found central for exercise hyperemia ${ }^{2}$ ); varying degrees of sympathetic dysfunction affecting blood pressure (BP); and vasoconstriction ${ }^{3}$ are suggested as contributing factors. The sum of factors results in blood to pool in the lower extremities and a smaller cardiac output. ${ }^{4}$ With this background, searching for effective training modalities for increasing cardiac output, peak oxygen uptake $\left(\mathrm{VO}_{2 \text { peak }}\right)$ and upper extremity work capacity for SCI is important. Presently, combining functional electrical stimulation (FES) in the lower extremities with upper extremity ACE or rowing (FES hybrid) is the preferred modality for training at the highest oxygen uptakes $\left(\mathrm{VO}_{2}\right) .{ }^{4,5} \mathrm{FES}$ activates the muscle venous pump, which increases venous return, end diastolic blood volume and preload to the left ventricle, increasing cardiac output and blood supply through the Frank-Starling mechanism, hence an improved upper extremity work capacity. ${ }^{3}$ FES hybrid cycling is resourcedemanding in terms of availability, cost and personal assistance. ${ }^{6}$ Furthermore, its use is mainly limited to those with an intact lower motor neuron and sensory motor complete injuries, because indivi- duals with preserved sensation below the level of injury have a lower tolerance for FES owing to various degrees of pain from stimulation. This calls for easier operable and affordable exercise training modalities.

One hypothesis is that applying leg vascular pressure in combination with ACE or wheelchair propulsion may facilitate venous return and further increase $\mathrm{VO}_{2 \text { peak }}$ in persons with SCI. ${ }^{7-9}$ Modalities applying compression, that is, anti-gravity suits (pressurized suits covering the lower extremities and the abdomen) with either pulsed or continuous pressure have been tested with conflicting results. ${ }^{8,10-12}$ Thus in the pursuit of a less resource-demanding training modality, applying leg vascular occlusion (LEVO) during ACE needs further assessment. As the rationale for applying LEVO during ACE is to facilitate venous return, effects will be addressed separately for SCI with level of injury above and below $\mathrm{T}_{6}$ (SCI-high and SCI-low, respectively). Individuals with SCI-high have a reduced sensory nervous system (SNS) outflow and supraspinal control to the splanchnic bed and lower extremity blood vessels, resulting in blood pooling during exercise. ${ }^{11}$ In persons with SCI-low injuries, the vasculature is generally innervated, especially in the important splanchnic vessels, and clinical manifestations of a circulatory SNS dysfunction (that is, venous pooling) during exercise may not be as marked as it is for individuals with high-level SCI. ${ }^{11}$ In this study our primary aim is to compare $\mathrm{VO}_{2 \text { peak }}$ between ACE, ACE combined with LEVO and FES hybrid cycling for individuals with SCI-high and SCI-low injuries.

${ }^{1}$ Department of Physical Medicine and Rehabilitation, St Olav's University Hospital, Trondheim, Norway; ${ }^{2}$ Faculty of Medicine, Department of Circulation and Medical Imaging, Norwegian University of Science and Technology, Trondheim, Norway; ${ }^{3}$ Faculty of Medicine, Department of Clinical Neuroscience, Norwegian University of Science and Technology, Trondheim, Norway and ${ }^{4}$ Hokksund Medical Rehabilitation Centre, Hokksund, Norway

Correspondence: B Brurok, Spinal Cord Injuries, St Olav's University Hospital, Olav Kyrres gt 17, Trondheim NO-7006, Norway.

E-mail: berit.brurok@ntnu.no

Received 21 March 2011; revised 17 October 2011; accepted 18 October 2011; published online 29 November 2011 
Our primary hypothesis is that ACE with LEVO results in higher $\mathrm{VO}_{2 \text { peak }}$ than ACE alone. Our secondary hypothesis is that FES hybrid cycling results in higher $\mathrm{VO}_{2 \text { peak }}$ compared with ACE with LEVO and ACE alone

\section{MATERIALS AND METHODS}

\section{Population and design}

We used a cross-sectional, single-subject design. Fifteen moderately active individuals with SCI were recruited from patient lists at the Department of Spinal Cord Injuries at St Olav's University Hospital, Norway.

Written informed consent was obtained from all individuals. They were divided into two groups: injuries above (SCI-high, $n=8$ ) and injuries below (SCI-low, $n=7$ ) $\mathrm{T}_{6}$ level.

The inclusion criteria were chronic/stable neurological state (at least 12 months since injury), paraplegia (lesion level between $\mathrm{T}_{1}$ and $\mathrm{L}_{1}$, where arm function is spared, but depending on the level of injury, the trunk, legs and pelvic organs may be involved) and tetraplegia (lesion level between $\mathrm{C}_{4}$ and $\mathrm{C}_{8}$, impairment of function in the arms as well as in the trunk, legs and pelvic organs), ${ }^{10}$ with lower motor neuron intact, ASIA Impairment Scale A and able to FES cycle for a minimum of1 min. Exclusion criteria were pacemakers, severe autonomic dysreflexia and decubitus in any area. Prior to inclusion an assessment consisting of neurological classification according to the ASIA Impairment Scale, skin health, resting BP and present cardiovascular disease in line with the recommendations from the $\mathrm{ACSM}^{13}$ was performed. Body mass in kilograms was obtained using an SECA digital chair scale 950 (Hamburg, Germany) calibrated for seated weighing. Individual characteristics are listed in Table 1.

\section{Test protocols}

We tested $\mathrm{VO}_{2 \text { peak }}$ during the three modalities in random order, on three separate days. All participants were familiar with ACE.

To determine $\mathrm{VO}_{2 \text { peak }}$, the following criteria had to be met: respiratory exchange ratio $(\mathrm{R}) \geqslant 1.05$, blood lactate level $\left(\left[\mathrm{La}^{-}\right]_{\mathrm{b}}\right)$ $\geqslant 7 \mathrm{mmol}$ and rating of perceived exertion (RPE) $\geqslant 15$, BORG scale (6-20). ${ }^{14}$ If these criteria were met, the average of the highest $\mathrm{VO}_{2}$ within three consecutive 10-s measurements was used to calculate $\mathrm{VO}_{2 \text { peak }}$.

\section{Apparatus}

Measurements of ventilatory parameters and pulmonary gas exchange were performed using a Metamax II Cortex ergospirometry system (Cortex Biophysik GmbH, Leipzig, Germany). Participants wore a facemask with a head cap assembly. A volume transducer was connected to the face mask with a tube that collects samples of the gas concentration in the mask every $10 \mathrm{~s}$. Prior to all tests calibration was

Table 1 Individual characteristics of the SCl-high and SCl-low groups

\begin{tabular}{lcc}
\hline & SCI-high $(\mathrm{n}=8)$ & SCl-low $(\mathrm{n}=7)$ \\
\hline TSI (years) & $12.9( \pm 10.8)$ & $13.5( \pm 11.7)$ \\
Weight (kg) & $73.3( \pm 8.4)$ & $74.9( \pm 14.9)$ \\
AIS & $\mathrm{A}$ & $\mathrm{A}$ \\
Level of injuries & $\mathrm{C}_{4}, \mathrm{C}_{5}, \mathrm{C}_{7}, \mathrm{C}_{7}, \mathrm{~T}_{2}, \mathrm{~T}_{5}, \mathrm{~T}_{5}, \mathrm{~T}_{5}$ & $\mathrm{~T}_{8}, \mathrm{~T}_{9}, \mathrm{~T}_{9}, \mathrm{~T}_{10}, \mathrm{~T}_{11}, \mathrm{~T}_{11}, \mathrm{~T}_{12}$ \\
Men/women & $8 / 0$ & $5 / 2$ \\
Age & $35( \pm 12.3)$ & $43.6( \pm 12.8)$
\end{tabular}

Abbreviations: AIS, ASIA Impairment Scale; C, cervical level; SCI, spinal cord injury; T, thoracic level; TSI, time since injury.

Data are presented as mean ( \pm s.d.). performed using a 3-1 standardized calibration syringe (Hans Rudolph Jäger GmbH, Kansas City, MO, USA). The gas concentration sensor was calibrated with ambient air and a chemically standardized calibration gas containing $16 \% \mathrm{O}_{2}, 4 \% \mathrm{CO}_{2}$ and $80 \%$ nitrogen (SensorMedics Corporation, Yorba Linda, CA, USA).

For ACE, the ERGOMED 840L (Siemens, München, Germany) was rebuilt and modified for ACE by changing the pedals for arm use (Figure 1).

For FES hybrid cycling the ERGYS 2 Rehabilitation System (Therapeutic Alliances Inc., Fairborn, OH, USA) was used (Figure 2).

For bilateral lower extremity vascular occlusion, single-hose thigh cuffs (NIBP; Criticare, Waukesha, WI, USA) were used.

Non-hemolysed blood lactate $\left(\left[\mathrm{La}^{-}\right]_{\mathrm{b}}\right)$ concentration was measured by using the portable Lactate Pro LT-1710 Analyzer (Arkray Factory Inc., KDK Corp., Kyoto, Japan). $\left[\mathrm{La}^{-}\right]_{\mathrm{b}}$ level was measured after all $\mathrm{VO}_{2 \text { peak }}$ tests in a capillary blood sample from a finger tip.

Heart rate (HR) was measured with Polar watches (Polar Electro, Oy, Finland). Peak HR was determined as the highest HR measured at

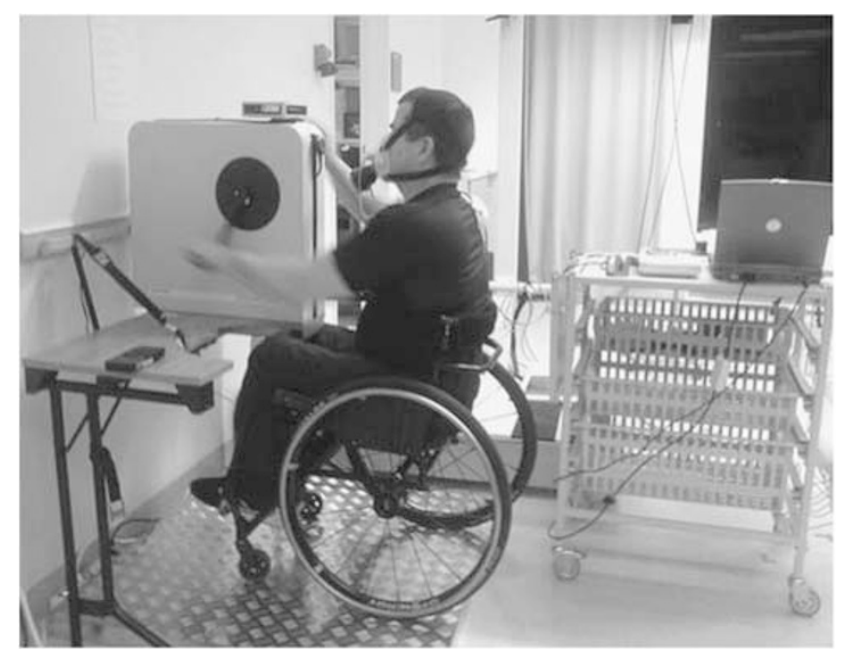

Figure 1 Setup for testing maximal oxygen uptake during ACE and ACE combined with LEVO.

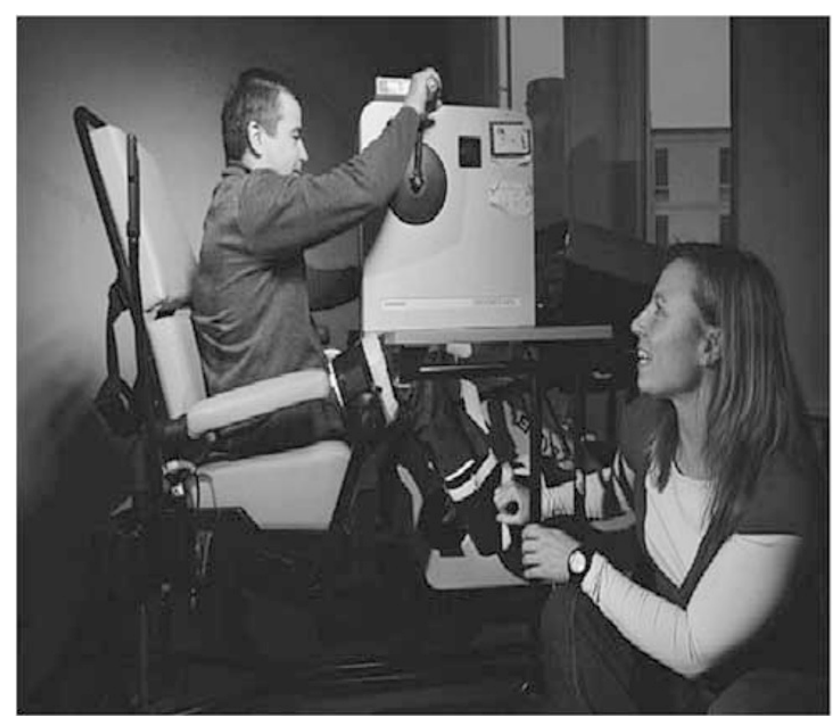

Figure 2 The FES hybrid cycling setup. 
the end of a $\mathrm{VO}_{2 \text { peak }}$ test. RPE (Borg 6-20) was recorded after each test. BP was measured by manual cuff and auscultation.

Oxygen pulse $\left(\mathrm{O}_{2}\right.$ pulse) was calculated as the ratio between $\mathrm{VO}_{2 \text { peak }}$ and peak HR.

\section{Test protocol for ACE}

During ACE the shoulder joint was horizontally aligned with the pedals and the elbows were positioned slightly flexed at the point of furthest reach. Tetraplegic individuals wore hand orthoses to enable performance during ACE. Four minutes of $30 \mathrm{~W}$ on the ACE were conducted directly followed by peak ACE, where ACE workload was increased each minute, tetraplegics by $5 \mathrm{~W}$ and paraplegics by $10 \mathrm{~W}$, until volitional fatigue. ACE peak Watt was determined by the highest Watt kept for the last $1 \mathrm{~min}$.

\section{Test protocol for ACE with LEVO}

Seated BP was measured at rest. Thigh cuffs were inflated to $100 \mathrm{~mm} \mathrm{Hg}$ above each individual's systolic BP as a tourniquet to achieve vascular hemostasis in the lower extremities. Then, $4 \mathrm{~min}$ of $\mathrm{ACE}$ at $30 \mathrm{~W}$ were conducted, directly followed by $\mathrm{VO}_{2 \text { peak }}$ test, where participants arm-cycled until volitional fatigue. The thigh cuffs were deflated immediately after the test.

\section{Test protocol for FES hybrid cycling}

The test began with 3 min of warm up on the ACE, followed by $4 \mathrm{~min}$ at $30 \mathrm{~W}$ on the ACE combined with 2 min of FES manual warm up, directly followed by the FES hybrid peak testing. No resistance was provided on the flywheel during FES cycling, whereas ACE workload was increased in 1 -min increments by $5-10 \mathrm{~W}$ until volitional fatigue. FES cycling started at individual time points, based on a pilot peak FES cycling test, to reach fatigue at approximately the same time for legs and arms. If FES cycling began to fatigue before the arms, the flywheel was manually assisted during FES leg cycling, to ensure stimulation current throughout the whole test. Self-adhesive electrodes were used and impulse frequency was set at $40 \mathrm{~Hz}$.

We certify that all applicable institutional and government regulations concerning ethical use of human volunteers were followed during the course of this research.

Statistical analyses. A Q-Q plot of $\mathrm{VO}_{2 \text { peak }}$ values did not reveal any indication for deviation from normal-distributed data. With two groups (SCI-high and SCI-low) and three modalities (ACE, ACE combined with LEVO and ACE combined with FES cycling) we used a two-by-three analysis of variance model for repeated measures. A $P$-value $<0.05$ was considered statistically significant (two-tailed). An interaction term was included to examine potential differences in $\mathrm{VO}_{2 \text { peak }}$ between type of exercise modality between SCI-high and SCI-low groups (full factorial model). Due to significant interaction, separate analyses for each group were performed, using repeatedmeasures analysis of variance, pairwise comparisons of peak oxygen uptake and related parameters between the three test modalities, with nominal significance level set to 0.017 to adjust for multiple comparisons (Bonferroni method).

\section{RESULTS}

\section{The SCI-high group}

For the SCI-high group mean $\mathrm{VO}_{2 \text { peak }}$ was $2.4 \mathrm{ml} \cdot \mathrm{kg}^{-1} \cdot \mathrm{min}^{-1}$ higher during ACE combined with LEVO compared with ACE alone (95\% confidence interval $(\mathrm{CI})$ : $0.8-3.9)(P=0.006)$; mean $\mathrm{O}_{2}$ pulse was 1.4-ml-beat ${ }^{-1}$ higher during ACE with LEVO compared with ACE alone $(P=0.046)(95 \% \mathrm{CI}$ : $0.024-2.7)$; and mean peak workload was $7.5 \mathrm{~W}$ higher during ACE combined with LEVO compared with ACE alone (95\% CI: 1.6-13.4) $(P=0.016)$. No differences in ventilation $\left(V_{E}\right),[L a]_{b}, R$, peak HR or RPE between these two modalities were found (Table 2).

FES hybrid cycling $\mathrm{VO}_{2 \text { peak }}$ was $4.4 \mathrm{ml} \cdot \mathrm{kg}^{-1} \cdot \mathrm{min}^{-1}$ higher $(95 \% \mathrm{CI}$ : 1.5-7.2) $(P=0.006)$ compared with ACE with LEVO. Mean $[\mathrm{La}]_{\mathrm{b}}$ was $1.9 \mathrm{mmol} \cdot \mathrm{l}^{-1}$ higher (95\% CI: $\left.0.7-3.0\right)(P=0.005)$ and mean $\mathrm{O}_{2}$ pulse was 1.9 ml.beat ${ }^{-1}$ higher (95\% CI: $\left.0.7-3.2\right)(P=0.005)$ during $\mathrm{FES}$ cycling compared with ACE with LEVO. No differences in R, peak HR or RPE between these two modes were observed.

$\mathrm{VO}_{2 \text { peak }}$ and workload during FES hybrid cycling compared with ACE alone were $6.8 \mathrm{ml} \cdot \mathrm{kg}^{-1} \cdot \mathrm{min}^{-1}$ and $10.5 \mathrm{~W}$ higher $(95 \% \mathrm{CI}$ : 3.5-10.0 $(P=0.001)$ and 95\% CI: $6.0-15.2(P=0.001)$, respectively). $\mathrm{V}_{\mathrm{E}}$ was $11.01 \cdot \mathrm{min}^{-1}$ higher $(95 \% \mathrm{CI}$ : $7.7-1.4)(P=0.000)$; $[\mathrm{La}]_{\mathrm{b}}$ was $1.9 \mathrm{mmol} \cdot \mathrm{l}^{-1}$ higher (95\% CI: $\left.0.2-3.5\right)$; $(P=0.029)$ and $\mathrm{O}_{2}$ pulse was $3.3 \mathrm{ml} \cdot$ beat $^{-1}$ higher (95\% CI: 1.6-5.0) $(P=0.001)$ during FES hybrid compared with ACE alone (Table 2).

\section{The SCI-low group}

$\mathrm{VO}_{2 \text { peak }}$ and related parameters were not different between ACE, ACE combined with LEVO and FES hybrid cycling. Peak HR was 5 beats min $^{-1}$ higher during ACE combined with LEVO compared with FES hybrid cycling (95\% CI: 1.6-8.9) $(P=0.009)$ (Table 3).

Table 2 Peak physiological parameters for the SCI-high group from the three test modalities

\begin{tabular}{|c|c|c|c|}
\hline Variables & $A C E(\mathrm{n}=8)$ & $A C E$ and $L E V O(\mathrm{n}=8)$ & FES hybrid cycling $(\mathrm{n}=8)$ \\
\hline \multicolumn{4}{|l|}{$\mathrm{VO}_{2 \text { peak }}$} \\
\hline$\left(I \cdot \min ^{-1}\right)$ & $1.24( \pm 0.40)$ & $1.48( \pm 0.43)^{*}$ & $1.80( \pm 0.40)^{\#, \S}$ \\
\hline$\left(\mathrm{ml} \cdot \mathrm{kg}^{-1} \cdot \mathrm{min}^{-1}\right)$ & $17.7( \pm 5.0)$ & $20.0( \pm 5.0)^{*}$ & $24.4( \pm 4.1)^{\#, \S}$ \\
\hline$V_{E}\left(I \cdot \min ^{-1}\right)$ & $50.4( \pm 20.8)$ & $54.3( \pm 22.7)$ & $61.4( \pm 19.8)^{\#, \S}$ \\
\hline $\mathrm{R}$ & $1.14( \pm 0.07)$ & $1.20( \pm 0.1)$ & $1.20( \pm 0.07)$ \\
\hline$\left[\mathrm{La}^{-}\right]_{\mathrm{b}}\left(\mathrm{mmol} \cdot \mathrm{I}^{-1}\right)$ & $7.5( \pm 1.1)$ & $7.5( \pm 1.2)$ & $9.4( \pm 1.7)^{\#, \S}$ \\
\hline $\mathrm{HR}_{\text {peak }}$ (beats. $\mathrm{min}^{-1}$ ) & $149( \pm 34)$ & $154( \pm 33)$ & $163( \pm 20)^{\S}$ \\
\hline Borg & $18( \pm 1)$ & $18( \pm 1)$ & $19( \pm 1)$ \\
\hline Watt & $72.5( \pm 32)$ & $80.0( \pm 34)^{*}$ & $83.0( \pm 32)^{\S}$ \\
\hline $\mathrm{O}_{2}$ pulse $\left(\mathrm{ml} \cdot\right.$ beats $\left.^{-1}\right)$ & $11.7( \pm 1.9)$ & $13.1( \pm 2.5)^{*}$ & $15.0( \pm 2.6)^{\#, \S}$ \\
\hline
\end{tabular}
Abbreviations: ACE, arm cycling; $\mathrm{HR}$, heart rate; $\left[\mathrm{La}^{-}\right]_{b}$, blood lactate; LEVO, lower extremity vascular occlusion; $\mathrm{O}_{2}$ pulse, oxygen pulse; $\mathrm{R}$, respiratory exchange ratio; SCI, spinal cord injury;

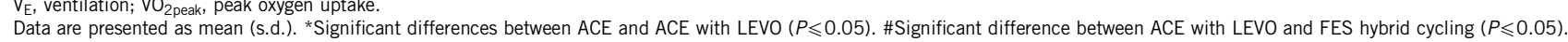
§Significant difference between ACE and FES hybrid cycling $(P \leqslant 0.05)$. 
Table 3 Peak physiological parameters for the SCl-low group from the three test modalities

\begin{tabular}{|c|c|c|c|}
\hline Variables & $A C E(\mathrm{n}=7)$ & ACE with LEVO $(\mathrm{n}=7)$ & FES hybrid cycling $(\mathrm{n}=7)$ \\
\hline \multicolumn{4}{|l|}{$\mathrm{VO}_{2 \text { peak }}$} \\
\hline$\left(I \cdot \mathrm{min}^{-1}\right)$ & $1.74( \pm 0.24)$ & $1.81( \pm 0.24)$ & $1.91( \pm 0.38)$ \\
\hline$\left(\mathrm{ml} \cdot \mathrm{kg}^{-1} \cdot \mathrm{min}^{-1}\right)$ & $23.7( \pm 3.6)$ & $24.4( \pm 3.7)$ & $25.6( \pm 4.1)$ \\
\hline $\mathrm{R}$ & $1.3( \pm 0.1)$ & $1.3( \pm 0.1)$ & $1.2( \pm 0.1)$ \\
\hline $\mathrm{V}_{\mathrm{E}}\left(1 \cdot \mathrm{min}^{-1}\right)$ & $76.6( \pm 13.4)$ & $80.8( \pm 11.5)$ & $80.2( \pm 21.1)$ \\
\hline$\left[\mathrm{La}^{-}\right]_{\mathrm{b}}\left(\mathrm{mmol} \cdot \mathrm{I}^{-1}\right)$ & $9.3( \pm 0.9)$ & $10.3( \pm 1.5)$ & $10.6( \pm 2.5)$ \\
\hline $\mathrm{HR}_{\text {peak }}$ (beats. $\mathrm{min}^{-1}$ ) & $185( \pm 11)$ & $187( \pm 9)$ & $182( \pm 9)^{\#}$ \\
\hline Watt & $96( \pm 23)$ & $100( \pm 22)$ & $98( \pm 19)$ \\
\hline Borg & $19( \pm 1)$ & $19( \pm 1)$ & $19( \pm 1)$ \\
\hline $\mathrm{O}_{2}$ pulse $\left(\mathrm{ml} \cdot\right.$ beat $\left.^{-1}\right)$ & $12.8( \pm 1.7)$ & $13.0( \pm 1.5)$ & $14.0( \pm 1.8)$ \\
\hline
\end{tabular}

Abbreviations: ACE, arm cycling; HR, heart rate; $\left[\mathrm{La}^{-}\right]_{b}$, blood lactate; LEVO, lower extremity vascular occlusion; $\mathrm{O}_{2}$ pulse, oxygen pulse; $\mathrm{R}$, respiratory exchange ratio; SCl, spinal cord injury; $\mathrm{V}_{\mathrm{E}}$, ventilation; $\mathrm{VO}_{2 \text { peak, }}$, peak oxygen uptake.

Data are presented as mean ( \pm s.d.). \#Significant difference between ACE with LEVO and FES hybrid cycling $(P \leqslant 0.05)$.

\section{DISCUSSION}

Our main finding is that LEVO in combination with ACE resulted in a $13 \%$ higher mean $\mathrm{VO}_{2 \text { peak }}$ compared with ACE alone for the SCI-high group, in line with our primary hypothesis. In the SCI-low group no difference was found between testing modalities. As a higher $\mathrm{VO}_{2 \text { peak }}$ from ACE combined with LEVO compared with ACE alone was found only in the SCI-high group, it may indicate that LEVO mainly augments the SNS reduced circulatory response to exercise as seen in individuals with SCI above $\mathrm{T}_{6}$. The rationale behind applying LEVO during ACE was to support blood redistribution, reduce lower extremity blood pooling and further improve upper extremity work capacity. The $12 \%$ higher $\mathrm{O}_{2}$ pulse (an indirect measure of stroke volume), together with the $10.3 \%$ higher peak workload and an insignificant difference in peak HR, may support this (Table 2). However, as heart function and blood pooling during testing were not measured in the present study, future randomized, controlled studies are needed to investigate if the underlying mechanism behind the increased $\mathrm{VO}_{2 \text { peak }}$ with $\mathrm{LEVO}$ is improved stroke volume owing to less lower body blood pooling and increased venous return. Previous studies have used leg positive pressure to augment $\mathrm{VO}_{2 \text { peak }}$ in SCI. Pitetti et al. ${ }^{8}$ found that a pulsating anti-gravity suit pressure of $50-75 \mathrm{~mm} \mathrm{Hg}$ in combination with ACE and/or wheelchair propulsion in eight tetraplegic individuals resulted in a higher mean $\mathrm{VO}_{2 \text { peak }}$ and arm work rate compared with arm exercise alone, in line with the findings for the SCI-high group in our study. Whereas Houtman et al. ${ }^{7}$ using a pulsating anti-gravity suit pressure in conjunction with ACE, assessing individuals with level of injury between $L_{1}$ and $L_{5}$, found no significant difference in $\mathrm{VO}_{2 \text { peak }}$ compared with ACE alone, similar to the findings for the SCI-low group in our study. However, comparisons between studies should be made with caution because of methodological diversity and population heterogeneity, that is, lack of consistent determination criteria for $\mathrm{VO}_{2 \text { peak }}$ and different levels of SCI. The criteria for $\mathrm{VO}_{2 \text { peak }}$ in our study differ from those by Pitetti et al. ${ }^{8}$ and Houtman et al. ${ }^{7}$ Furthermore, creating group homogeneity according to level and completeness of SCI, age and gender is challenging, although important when assessing aerobic performance.

In the SCI-high group, two of the tetraplegic individuals did not satisfy the criteria for peak levels of blood lactate during ACE and ACE with LEVO (6.7 and 5.7, and 5.0 and $6.7 \mathrm{mmol} \cdot \mathrm{l}^{-1}$, respectively). This may be because they ended the tests owing to hypotension from sitting up and exercising. However, high values for R and RPE suggest that they exercised at maximal effort during both modalities; thus they were included in the analysis. An overestimation of the effect from LEVO in the SCI-high group is unlikely, as the determination criteria

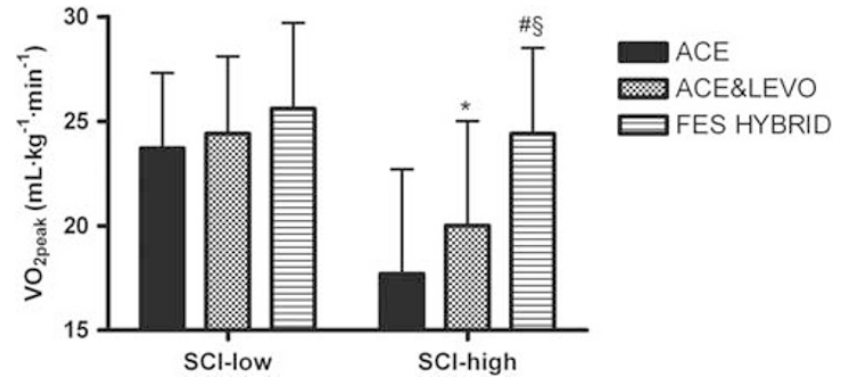

Figure 3 Maximal oxygen uptake measured for the $\mathrm{SCl}$-high and $\mathrm{SCl}$-low groups during ACE, ACE combined with LEVO and FES leg cycling combined with ACE. Data are presented as mean \pm s.d. ACE, arm cycling; LEVO, leg vascular occlusion; FES hybrid, functional electrical cycling combined with arm cycling; $\mathrm{VO}_{2 \text { peak, }}$ peak oxygen uptake. *Significant difference between ACE and ACE with LEVO. \#Significant difference between ACE with LEVO and FES hybrid cycling. §Significant difference between ACE and FES hybrid cycling $(P \leqslant 0.05)$.

for $\mathrm{VO}_{2 \text { peak }}$ were not reached during both modalities. The same two individuals were also, because of exercise-induced hypotension, unable to perform the total of $4 \mathrm{~min}$ of ACE warm up at $30 \mathrm{~W}$, but were able to perform ACE warm up with LEVO. Therefore, one may speculate that LEVO facilitates exercising without a reduction in BP; however as $\mathrm{BP}$ was not measured during exercise, future studies are needed to assess this.

LEVO during ACE increased $\mathrm{VO}_{2 \text { peak }}$ in the SCI-high group; however it was still below the $\mathrm{VO}_{2 \text { peak }}$ levels achieved during FES hybrid testing, in line with our hypothesis (Figure 3). Compared with LEVO, FES cycling recruits more muscle mass and activates the muscle venous pump. An increase of muscle mass during FES hybrid testing will increase oxygen consumption through increased mitochondrial activity together with a higher venous return and stroke volume, hence a higher $\mathrm{VO}_{2 \text { peak }}$ is likely reached. ${ }^{15}$ In contrast to our hypothesis, no difference in $\mathrm{VO}_{2 \text { peak }}$ between the three test modalities was found for the SCI-low group. Thus it appears that SNS innervation has an important role in the redistribution of blood during exercise.

Although the effect from LEVO on $\mathrm{VO}_{2 \text { peak }}$ is lower than during FES hybrid cycling, it may serve as a less resource-demanding alternative to ACE alone for persons with SCI-high. Even small increases in $\mathrm{VO}_{2}$, especially in persons with tetraplegia, may translate into improvements in their ability to perform activities of daily living and provide additional energy to participate in social life. ${ }^{16}$ That is, the metabolic cost of activities of daily living in persons with complete 
SCI is $2.7 \mathrm{ml} \cdot \mathrm{kg}^{-1} \cdot \mathrm{min}^{-1}$ compared with $3.5 \mathrm{ml} \cdot \mathrm{kg}^{-1} \cdot \mathrm{min}^{-1} \mathrm{O}_{2}$ for able-bodied persons; ${ }^{17}$ and the SCI metabolic cost, that is, during deskwork is 1.47; during dusting is 1.27; and during wheeling on grass is $4.96 .{ }^{17}$ In comparison, the metabolic cost of ACE in the present study is 6.4, ACE with LEVO is 7.4 and FES hybrid cycling is 9.0, highlighting the benefit from the three tested modalities. Follow-up studies of able-bodied persons have found that $\mathrm{VO}_{2 \text { peak }}$ is highly associated with morbidity and mortality, ${ }^{18,19}$ indicating the importance of $\mathrm{VO}_{2 \text { peak }}$ as a prognostic factor and a measure of overall cardiovascular health. This may further reflect the need for finding easy accessible training modalities and the most effective training intensity to reach the highest possible exercise oxygen uptake for SCI.

The SCI-high group included both tetraplegic and paraplegic individuals. Ideally, as they elicit different physiological exercise responses, they should have been separated into different groups. However, we considered lack of SNS innervation to the splanchnic vascular bed and lower extremity blood vessels, and a low number of participants, more important than innervation to the heart and lack of small muscles in the hand, and therefore split the groups at the $\mathrm{T}_{6}$ level.

It is of interest to compare $\mathrm{VO}_{2 \text { peak }}$ and related parameters between the two groups; however we believe that the combination of a small number of participants and the relatively large differences in mean age between the groups made it difficult to separate the effects of age from the effects of SCI-high versus SCI-low. A 10-year age difference is likely to have a significant effect on aerobic performance between the groups. Furthermore, as $\mathrm{VO}_{2 \text { peak }}$ does not represent an effect from each test modality owing to lack of baseline values, the most reasonable focus in the present study was the difference in $\mathrm{VO}_{2 \text { peak }}$ from the training modalities within the SCI-high and SCI-low groups separately. Therefore, future randomized, controlled studies where groups are matched for age and gender are needed to assess betweengroup differences.

Thigh cuff pressures during LEVO in this study were individually set to $100 \mathrm{~mm} \mathrm{Hg}$ above systolic BP. This is high compared with the anti-gravity suit pressures used in previous studies, ${ }^{8,9,12}$ but lower than the pressure used in the study by Kjar et al. ${ }^{20}$ In the surgical setting, occlusion usually extends to 1 to $1 \frac{1 / 2}{h}$, but in the present study it extended only for a maximum time of $10 \mathrm{~min}$, lowering the risk of possible tissue damage. ${ }^{21}$ These pressures will inflict arterial vessels and may cause ischemic damage, but no reports of adverse events related to vascular pressures are given in similar studies..$^{20}$

\section{Study limitations}

This study is limited as blood pooling, heart function and BP were not measured, and thus the mechanisms behind the increase in $\mathrm{VO}_{2 \text { peak }}$ in the present study remain unknown. Furthermore, a low number of participants and lack of homogeneity in the groups reduce the statistical power and make it difficult to generalize the findings.

\section{Conclusions}

Applying LEVO during ACE for the SCI-high group in the present study augmented $\mathrm{VO}_{2 \text { peak }}$ compared with ACE alone, but not to the level of the FES hybrid mode. No effect from LEVO was found for the SCI-low group. ACE combined with LEVO may serve as a less resourcedemanding alternative to FES hybrid, for those with high-level SCI in the present study. These findings may have future implications for exercise prescription and cardiovascular prevention programs.

\section{CONFLICT OF INTEREST}

The authors declare no conflict of interest.

\section{ACKNOWLEDGEMENTS}

We acknowledge the participation of the SCI individuals; Professor Jon Magnussen at the Department of Public Health and General Practice, NTNU, for timely and critical reading of the manuscript; and Professor Grethe Albrektsen at the Department of Cancer Research and Molecular Medicine, Unit for Applied Medical Research, NTNU, for valuable help with the statistical analyses. This work was supported by St Olav's Hospital-Trondheim University Hospital, Liaison Committee between the Central Norway Regional Health Authority and the Norwegian University of Science and Technology, and SINTEF Unimed.

1 Myers J, Lee M, Kiratli J. Cardiovascular disease in spinal cord injury: an overview of prevalence, risk, evaluation, and management. Am J Phys Med Rehabil 2007; 86: $142-152$.

2 Hamann JJ, Valic Z, Buckwalter JB, Clifford PS. Muscle pump does not enhance blood flow in exercising skeletal muscle. J Appl Physiol 2003; 94: 6-10.

3 Phillips WT, Kiratli BJ, Sarkarati M, Weraarchakul G, Myers J, Franklin BA et al. Effect of spinal cord injury on the heart and cardiovascular fitness. Curr Probl Cardiol 1998; 23: 641-716.

4 Hooker SP, Figoni SF, Rodgers MM, Glaser RM, Mathews T, Suryaprasad AG et al. Metabolic and hemodynamic responses to concurrent voluntary arm crank and electrical stimulation leg cycle exercise in quadriplegics. J Rehabil Res Dev 1992; 29: 1-11.

5 Brurok B, Helgerud J, Karlsen T, Leivseth G, Hoff J. Effect of aerobic high-intensity hybrid training on stroke volume and peak oxygen consumption in men with spinal cord injury. Am J Phys Med Rehabil 2011; 90: 407-414.

6 Donovan-Hall MK, Burridge J, Dibb B, Ellis-Hill C, Rushton D. The views of people with spinal cord injury about the use of functional electrical stimulation. Artif Organs 2011; 35: 204-211.

7 Houtman S, Thielen JJ, Binkhorst RA, Hopman MT. Effect of a pulsating anti-gravity suit on peak exercise performance in individual with spinal cord injuries. Eur J Appl Physiol Occup Physiol 1999; 79: 202-204.

8 Pitetti KH, Barrett PJ, Campbell KD, Malzahn DE. The effect of lower body positive pressure on the exercise capacity of individuals with spinal cord injury. Med Sci Sports Exerc 1994; 4: 463-468.

9 Kaprielian R, Plyley MJ, Klentrou P, Goodman LS, Goodman JM. The effect of lower body positive pressure on the cardiovascular response to exercise in sedentary and endurance-trained persons with paraplegia. Eur J Appl Physiol 1998; 78: 141-147.

10 Mulcahey MJ, Gaughan J, Betz RR, Johansen KJ. The international standards for neurological classification of spinal cord injury: reliability of data when applied to children and youths. Spinal Cord 2006; 45: 452-459.

11 Teasell RW, Arnold JMO, Krassioukov A, Delaney GA. Cardiovascular consequences of loss of supraspinal control of the sympathetic nervous system after spinal cord injury. Arch Phys Med Rehabil 2000; 81: 506-516.

12 Hopman MT, Kamerbeek IC, Pistorius M, Binkhorst RA. The effect of an anti-G suit on the maximal performance of individuals with paraplegia. Int J Sports Med 1993; 14: 357-361.

13 ACoS Medicine. ACSM's Guidelines for Exercise Testing and Prescription, 8th edn. Wolters Kluwer Health Ltd, Pennsylvania, PA, USA, 2009.

14 Åstrand PO, Rodahl K, Dahl HA, Strømme SB. Textbook of Work Physiology: Physiological Bases of Exercise, 4th edn. McGraw-Hill: New York, 2003.

15 McArdle WD, Katch F, Katch VL. Exercise Physiology Energy, Nutrition, and Human Performance. 5th edn Lippincott Williams \& Wilkins: Philadelphia, 2001.

16 Price M. Energy expenditure and metabolism during exercise in persons with a spinal cord injury. Sports Med 2010; 40: 681-696.

17 Collins EG, Gater D, Kiratli J, Butler J, Hanson K, Langbein WE. Energy cost of physical activities in persons with spinal cord injury. Med Sci Sports Exerc 2010; 42: 691-700.

18 Keteyian SJ, Brawner CA, Savage PD, Ehrman JK, Schairer J, Divine G et al. Peak aerobic capacity predicts prognosis in patients with coronary heart disease. Am Heart $J$ 2008; 156: 292-300.

19 Myers J, Prakash M, Froelicher V, Do D, Partington S, Atwood JE. Exercise capacity and mortality among men referred for exercise testing. N Engl J Med 2002; 346: 793-801.

20 Kjar M, Pott F, Mohr T, Linkis P, Tornoe P, Secher NH. Heart rate during exercise with leg vascular occlusion in spinal cord-injured humans. J Appl Physiol 1999; 86: 806-811.

21 Tejwani NC, Immerman I, Achan P, Egol KA, McLaurin T. Tourniquet cuff pressure: the gulf between science and practice. Journal Trauma 2006; 61: 1415-1418. 